\title{
Sociedad, epistemología y metodología en Boaventura de Sousa Santos
}

\author{
Society, epistemology and methodology in Boaventura de Sousa Santos
}

\author{
Helder Binimelis-Espinoza / hbinimelis@uct.cl \\ orcid.org/0000-0001-5626-0109 \\ Universidad Católica de Temuco, Chile
}

Andrés Roldán-Tonioni / roldantonioni.andres@outlook.es

orcid.org/0000-0001-7938-2854

Universidad de Chile, Chile

\begin{abstract}
The object of this essay is to analyze the work of Boaventura de Sousa Santos, especially his understanding of contemporary societies and today's epistemological and methodological challenges. Based on a documentary review of his work and critical contributions by other authors, we distinguish his critical utopian understanding of today's societies, and the need to renew the processes of knowledge construction. On the one hand, this work integrates Santos' criticism of modernity with his utopian proposal and his relations with southern hemisphere epistemologies, the ecology of knowledge and intercultural translation. On the other, it identifies concrete strategies for knowledge generation from an intercultural perspective. The conclusions are oriented toward a critical assessment of the discussion developed with Santos, and its relevance in the context of the social sciences in Latin America.
\end{abstract}

Key words: social regulation, social emancipation, utopia, epistemologies of the south, methodology.

Resumen: El objetivo de este ensayo es analizar la obra de Boaventura de Sousa Santos, especialmente su comprensión de las sociedades contemporáneas y los desafíos epistemológicos y metodológicos actuales. Mediante una revisión documental de su obra y aportes críticos de otros autores, se distingue su comprensión crítica y utópica de las sociedades actuales, y la necesidad de renovación de los procesos de construcción de conocimiento. Los aportes de este trabajo son, por una parte, integrar la crítica de Santos a la modernidad con su propuesta utópica, y su relación con las epistemologías del sur, la ecología de saberes y la traducción intercultural. Por otra parte, la identificación de estrategias concretas de generación de saberes desde una perspectiva intercultural. Las conclusiones están orientadas hacia una evaluación crítica de la discusión desarrollada con Santos y su relevancia en el contexto de las ciencias sociales latinoamericanas.

Palabras clave: regulación social, emancipación social, utopía, epistemologías del sur, metodología. 


\section{Introducción}

A partir de la propuesta teórica de Santos, es posible identificar tres grandes desafíos para las ciencias sociales. 1) La comprensión de sociedades que desarrollan complejos procesos institucionales y de relaciones entre actores sociales locales, nacionales y globales (Santos, 1998a, 2003 y 2010). 2) Los problemas epistemológicos del diálogo intercultural con otros saberes, con concepciones de mundo más allá del eurocentrismo y de la racionalidad científica dominantes (Santos, 2005 y 2006). 3) Los desafíos epistemológicos y metodológicos orientados por la crítica y la intención de producir transformaciones sociales (Santos, 2009).

Estos desafíos se asumen en un contexto poco favorable, pues las sociedades capitalistas promueven mecanismos de regulación social que deben ser desvelados por medio de la reflexión y la acción crítica. En la medida que la propia ciencia se ha constituido en uno de estos mecanismos regulatorios, es necesario buscar más allá de sus fronteras. Es ineludible indagar en comprensiones de mundo y de producción de conocimiento que puedan orientar unas ciencias sociales críticas que acojan tanto los nuevos problemas del mundo globalizado como la necesidad del diálogo intercultural.

El diálogo crítico con la propuesta teórica y epistemológica de Boaventura de Sousa Santos parece fundamental para discutir sobre estas cuestiones, en la medida en que se ha convertido en un referente para las ciencias sociales y el pensamiento crítico contemporáneo. Por una parte, es posible observar un creciente uso de las construcciones conceptuales del pensador portugués en relación con investigaciones en diversos ámbitos de las ciencias sociales y las humanidades, como por ejemplo en los ámbitos de educación (Passos, 2014; Baker, 2012; Streck y Adams 2012; Marques, 2008; Anibal, 2010), derecho (Umut y Ballesteros, 2014; Barreto, 2014; Gutierrez 2013; Sieder, 2011; Álvarez, 2010; Guarino, 2009) y sociología (Santos et al., 2015; Paredes, 2014; Machado et al., 2012). Esta influencia abarca también investigaciones de corte histórico (Sarmento, 2015) y de estudios sociales sobre salud (Nascimento et al., 2013).

En todos los casos anteriores la propuesta de Santos es adaptada a lógicas de investigación críticas, pero que no explicitan estrategias de diálogo con saberes y prácticas surgidas de luchas emancipatorias concretas.

Por otra parte, hay una búsqueda de profundizar en diversos aspectos de su obra. Pueden destacarse los trabajos de Aguiló (2011) sobre la concepción de Santos sobre globalización y diálogo intercultural; el de Pereira y Carvalho (2008) que busca desentrañar su concepción de democracia y democracia 
participativa; el de Villareal (2006), donde analiza la relación que éste hace entre ciudadanía y emancipación; o la crítica de Darian-Smith (1998) a la concepción de utopía del portugués. Destaca el aporte de Avritzer (2002), quien establece una comparación de Santos con Habermas y Giddens, respecto a cómo estos autores abordan el problema de la modernidad y de la ciudadanía en el contexto de la globalización. Por su parte, el trabajo de Tamayo (2011) en el cual propone una revisión de los desafíos epistemológicos.

El presente documento busca tender puentes entre lo que consideramos los elementos centrales de la propuesta de Santos, es decir, su crítica a la modernidad, el fundamento utópico de esa crítica, y los desafíos epistemológicos que esto trae consigo; y la identificación de estrategias metodológicas concretas para la generación de saberes desde una perspectiva intercultural, buscando distinguir algunos de los elementos que lo diferencian de las ciencias sociales convencionales.

Se abordará, en primer lugar, su crítica al proyecto de la modernidad como regulación social, en la cual se ha reducido el proyecto amplio de la modernidad en los particularismos del capitalismo. En segundo lugar, esta comprensión de la sociedad será confrontada con la noción de utopía como fundamento de la transición paradigmática en curso. En tercer lugar, se presentarán los principales elementos de la denominada Epistemología del Sur, para avanzar en cuarto lugar con los principales elementos metodológicos, es decir, con el desarrollo práctico de procesos de producción de saber, orientados por la utópica búsqueda de emancipación social de las sociedades capitalistas, considerando los desafíos interculturales que esta tarea trae consigo.

\section{Modernidad regulatoria y crisis global}

La modernidad, un proyecto originalmente emancipatorio, convergió en un único proyecto de regulación económico, político y cultural-civilizatorio: el capitalismo . Con ello, los procesos emancipatorios fueron relegados o eliminados.

A través de la función de regulación ejercida por los Estados nacionales y los mercados capitalistas, la desigualdad y la exclusión pasaron de ser excepcionales a constantes, convirtiéndose en sistemas de jerarquización de las sociedades modernas; es decir, mecanismos que regulan la desigualdad mediante la integración subordinada, y mecanismos que regulan la exclusión mediante la integración excluyente (Santos, 2005).

Según Santos (2005), las principales luchas sociales, políticas, económicas y culturales durante la modernidad han estado asociadas a la tensión 
entre regular la desigualdad y la exclusión, o por el contrario, a la búsqueda de emancipación respecto a estos sistemas de pertenencia jerarquizados. Avritzer (2002) considera que las sociedades modernas viven una constante tensión dialéctica entre regulación y emancipación, que se manifiesta más allá de los espacios de acción del capital y del Estado.

La legitimación de estos sistemas de regulación en las sociedades modernas, además de basarse en visiones esencialistas, ideologías y prácticas cotidianas, se ha cristalizado en las justificaciones provistas por la ciencia moderna y el derecho, tanto por su subordinación a principios políticos y culturales nacionales, como por su vinculación creciente con procesos económicos capitalistas, y por ello, con fuerte vinculación con las diversas formas de poder existentes en sociedad (Santos, 2003 y 2009). Se plantea aquí que la distinción de estas diversas formas de regulación (desde la regulación social moderna hasta la regulación del conocimiento) es una de las claves interpretativas de la obra de Santos y su búsqueda plural de emancipación.

En este contexto, la exclusión se convierte en una nueva frontera para la desigualdad económica. En primer lugar, los cambios productivos del capitalismo, que implican la transformación global del trabajo, la flexibilización de la producción, un nuevo paradigma tecno-económico, la ampliación de los mercados financieros, la aparición de bloques comerciales y la legitimación de "zonas especiales" con reglas del juego premodernas (maquilas, paraísos fiscales, etcétera).

En segundo lugar, la aparición de recurrentes crisis en el espacio de los Estados Nacionales, que al depender de precios internacionales, de políticas fiscales y monetarias impuestas, orientadas a privatizar y reducir los espacios públicos, a eliminar las regulaciones sobre el trabajo y el control estatal sobre la economía, no pueden responder adecuadamente a las demandas de empleo y consumo, y, por tanto, tienen menos posibilidades de proponer y cumplir pactos sociales que los hagan viables (Santos, 2005).

Santos (1998a: 371) señala que, dentro del orden social moderno, no es posible encontrar respuestas a las mencionadas crisis, debido a la imposibilidad de resolver los denominados problemas fundamentales, es decir, aquellos que:

[...] están en la raíz de nuestras instituciones y de nuestras prácticas, modos profundamente arraigados de estructuración y de acción sociales considerados por algunos como fuentes de contradicciones, antinomias, incoherencias, injusticias, que repercuten con intensidad variable en los más diversos sectores de la vida social. Tales repercusiones son acumulativas, por lo que son vistas en proceso de empeoramiento continuo y con la posibilidad de desenlaces más o menos críticos a mediano o a largo plazo. 
Frente a ello, Santos (2003) propone la revalorización y primacía de la emancipación tanto de los diversos espacios sociales como de las diversas formas de pensamiento. Implica restablecer el equilibrio entre regulaciónemancipación, superando los límites impuestos por las sociedades modernas. La cuestión fundamental es, entonces, el desarrollo de una estrategia que permita la transición paradigmática de la regulación a la emancipación. Esta búsqueda implica otro aspecto central de la propuesta de Santos: la recuperación crítica y posmoderna de la utopía.

\section{Una utopía posmoderna y de oposición}

La utopía de Santos posee una dimensión moderna, pero también una posmoderna. La primera dimensión lo conecta con aquellas facetas de la modernidad olvidadas o postergadas y que no se convirtieron en mecanismos de regulación. La segunda, con el reconocimiento de la crisis social actual:

[...] el posmodernismo de oposición, se distingue a sí mismo tanto de la modernidad como del posmodernismo celebratorio por el reconocimiento, contra la modernidad, de la profundidad de los cambios en curso (la crisis final de la modernidad), y al insistir, contra el posmodernismo celebratorio en la persistencia de la invarianza que de hecho generan los mencionados cambios (el capitalismo global). Esta es la razón por la que la reconstrucción emancipatoria de los cambios basada en la superación de la invarianza, solo será posible en las actuales circunstancias teniendo como objetivo una aspiración utópica (Santos, 1998b: 138. Traducción propia).

Darian-Smith (1998) desarrolla una crítica a la visión utópica de Santos que puede resumirse así: Si la utopía es una concepción de mundo moderna, ¿no implicaría que como solución a la crisis de la modernidad caería en los mismos errores que otras alternativas de la modernidad regulatoria? Como la utopía es moderna, ¿será igualmente incapaz de responder a los problemas fundamentales? Por ello, es conveniente distinguir cuáles son los nuevos componentes posmodernos críticos que le dan un nuevo sentido a la utopía como camino de salida de la crisis social actual.

La utopía moderna nos propone algo que no existe, pero que nos orienta a la reconstrucción de nuestro futuro. Por su parte, Santos (2003: 378) propone la recuperación de aquello que ha sido declarado como inexistente o que ha sido silenciado por los mecanismos regulatorios:

Mi versión de la utopía es, pues, doblemente relativa. Por un lado, llama la atención sobre lo que no existe en tanto que (contra) parte integrante, aunque silenciada de aquello que existe, o sea, sobre aquello que pertenece a una determinada época por el modo como está excluido de ella. Por otro lado, la utopía siempre es desigualmente 
utópica, dado que su forma de imaginar lo nuevo está parcialmente constituida por nuevas combinaciones y escalas de aquello que existe, y que son, en verdad, casi siempre pormenores, pequeños y oscuros, de lo que realmente existe. La utopía requiere, por consiguiente, un conocimiento abarcador y profundo de la realidad como medio para evitar que el radicalismo de la imaginación colisione con su realismo.

El espacio de la utopía moderna es el Estado y la organización sociopolítica de la sociedad. La utopía posmoderna, junto con el proyecto político (la refundación de las relaciones Estado-sociedad), propone utopías para la vida familiar, sexual y doméstica, respecto a formas de producción eco-socialistas, a un consumo orientado por necesidades humanas, a prácticas comunitarias, identitarias e interculturales orientadas por la búsqueda de la dignidad humana, y unas relaciones globales cosmopolitas y antihegemónicas.

En esta apertura de la utopía, más allá del Estado, se pone de manifiesto una comprensión de la sociedad que rompe con un orden estructural cerrado, donde únicamente el Estado es un agente de poder y dominación, cuando, por el contrario, estos procesos (y las búsquedas de emancipación) se ponen de manifiesto en diversos espacios de acción cotidianos, desde el trabajo y el consumo, hasta el racismo y la vida íntima (Santos, 2003).

Por otra parte, la modernidad y la posmodernidad celebratoria han establecido límites regulatorios a la utopía. Dentro de sus márgenes, sólo se la puede pensar en términos morales e ideales (Santos, 2003). En este sentido se plantea la desacreditación del pensamiento utópico. Santos piensa la utopía en oposición a la regulación y el realismo de la ciencia y el derecho. La utopía se plantea como precaución frente a la posibilidad que un proyecto emancipatorio tienda nuevamente a la regulación produciendo nuevas desigualdades, nuevas exclusiones, nuevos silenciamientos.

La utopía posmoderna se opone a la alienación de las subjetividades individuales y colectivas, encerradas en los límites de la modernidad regulatoria, incapaces de plantearse la emancipación social como algo factible de ser realizado. Se plantea como la posibilidad de ampliar los espacios de acción democráticos hacia aquellos lugares que la modernidad ha considerado hasta el momento irrelevantes.

Este nuevo proyecto utópico posmoderno y crítico requiere una doble transformación para su gradual consolidación o, como expresa Santos (1998a: 426), requiere de dos "condiciones de posibilidad". Por una parte, una nueva forma de conocimiento, una nueva epistemología, y por otra, una nueva subjetividad individual y colectiva; es decir, comunidades e individuos que más allá de los límites modernos del realismo científico crean en la posibilidad de emancipación (Santos, 2003). 
Helder Binimelis-Espinoza y Andrés Roldán-Tonioni. Sociedad, epistemología y metodología en Boaventura de Sousa Santos

\section{Sobre la Transición paradigmática hacia una Epistemología del Sur}

Las crisis fundamentales de las sociedades actuales tienen su manifestación específica en la generación de conocimiento científico reducido a una forma de regulación moderna. En los inicios de la modernidad existía un equilibrio dinámico entre las dos formas de conocimiento modernas: el conocimiento-regulación y el conocimiento-emancipación. Este equilibrio se mantenía a través de tres lógicas de racionalidad: la moral práctica, la estético-expresiva y la cognitivo-instrumental (Santos, 2006 y 2003). La reducción de la modernidad a un proyecto regulatorio implicó la absorción del conocimiento-emancipación por la regulación: "La colonización gradual de las diferentes racionalidades de la emancipación moderna por la racionalidad cognitivo-instrumental de la ciencia subsumió la concentración de las energías y de las potencialidades emancipadoras de la modernidad en la ciencia y en la técnica" (Santos, 2003: 59-60).

La hegemonía de la regulación orienta los proyectos emancipatorios hacia un orden científico-técnico que transforma toda forma de conocimiento contraria al orden en caos, y todo conocimiento contrario a la regulación, en ignorancia (Santos, 2009). Las expresiones de esta falta de reconocimiento de otros saberes son el colonialismo como forma dominante de conocimiento regulador; y el epistemicidio, que implica la negación y destrucción de saberes contrarios al orden dominante (Santos, 1998a).

El privilegio epistemológico que la ciencia moderna se concede a sí misma es, pues, el resultado de la destrucción de todos los conocimientos alternativos que podrían venir a enjuiciar ese privilegio. En otras palabras, el privilegio epistemológico de la ciencia moderna es producto de un epistemicidio. La destrucción del conocimiento no es un artefacto epistemológico sin consecuencias, sino que implica la destrucción de prácticas sociales y la descalificación de agentes sociales que operan de acuerdo con el conocimiento enjuiciado (Santos, 2009: 81).

La ciencia reguladora se ha convertido en una práctica de generación de conocimiento, que al negar la validez de otras formas de producción de saber, se configura como el punto de vista y discurso privilegiado. Sólo ese "espectador ideal" en quien se constituye el científico puede obtener esta prerrogativa (Santos, 2009: 70).

Un aspecto central de la actual crisis de la modernidad tiene que ver con la extensión de las consecuencias de la ciencia y la técnica más allá de las posibilidades de previsión establecidas dentro del orden moderno. El pensador portugués señala que las escalas espaciales y temporales en que la ciencia y la tecnología afectan la sociedad convierte los nexos causales en opacos y 
complejos; es decir, las consecuencias de la acción científica se alejan de las pretensiones originales de cientificidad, y se vuelven incontrolables.

La promesa de una paz perpetua, basada en el comercio, en la racionalización científica de los procesos de decisión y de las instituciones, condujo al desarrollo tecnológico de la guerra y al aumento sin precedentes de su poder destructivo. La promesa de una sociedad más justa y libre, sustentada en la creación de riqueza fue hecha posible por la conversión de la ciencia en fuerza productiva, condujo a la expoliación del Tercer Mundo y a un abismo cada vez mayor entre el Norte y el Sur (Santos, 2003: 60).

Contradictoriamente, una ciencia que pueda proyectarse en resultados previsibles y transformaciones sociales de gran escala queda atada a la inmediatez de la utilidad (Santos, 2003). Por ello, la transición paradigmática respecto al conocimiento regulación implica prestar atención a las lógicas de racionalidad dejadas de lado por la modernidad capitalista, y en específico, a las lógicas de racionalidad menospreciadas o destruidas por la ciencia reguladora.

En contraste con la posición de privilegio que la ciencia convencional establece para sí misma, el nuevo paradigma emancipador tiene como punto de partida las experiencias prácticas de sufrimiento. No es posible generalizarlo a través de un modelo preestablecido y permanente. En este sentido, adquiere especial importancia la recuperación de experiencias vitales históricamente postergadas o "silenciadas", aquellas que han logrado sobrevivir marginalmente, y aquellas que emergen frente a los nuevos problemas actuales. Es aquí donde adquiere especial relevancia la metáfora sobre el Sur, que toma como modelo la propuesta de Edward Said (2007) respecto al orientalismo. Así como el Oriente ha sido construido en contraposición a las sociedades occidentales, el Sur representa la subordinación al Norte. El desafío en relación con el conocimiento implica:

[...] la búsqueda de conocimientos y de criterios de validez del conocimiento que otorguen visibilidad y credibilidad a las prácticas cognitivas de las clases, pueblos y de los grupos sociales que han sido históricamente victimizados, explotados y oprimidos por el colonialismo y capitalismo globales (Santos, 2009: 12).

Lo que daría sustento a esta nueva epistemología es, por tanto, una forma de conocimiento que se oriente por aquello que en la modernidad capitalista ha sido postergado y silenciado, la solidaridad: "La solidaridad como forma de conocimiento es el reconocimiento del otro como igual, siempre que la diferencia le acarree inferioridad; y como diferente, siempre que la igualdad le ponga en riesgo la identidad" (Santos, 2009: 87).

Esto implica asumir una actitud prudente frente a los resultados, las expectativas, y no una actitud orientada por el triunfo del progreso y la utilidad 
económica. La prudencia es el control de la confianza y el control de las relaciones con el poder; es decir, la actitud crítica de estar atentos a la posibilidad de desarrollar voluntaria o involuntariamente mecanismos de subordinación (Santos, 2003: 2009).

En vez de buscar verdades irrefutables, el nuevo paradigma se orienta por la construcción argumentativa del conocimiento; es decir, por causas e intenciones situadas y orientadas en relación con audiencias específicas; por lo tanto, la legitimación de un conocimiento nunca implica aceptar verdades intemporales e incuestionables. Santos (1998a: 433) señala que: "El conocimiento del nuevo paradigma no es validable por principios demostrativos de verdades intemporales. Es, por el contrario, un conocimiento retórico cuya validez depende del poder de convicción de los argumentos en que se traduce".

Santos (1998a) utiliza el concepto de preclusión, término de origen jurídico que implica la pérdida de una capacidad; en este caso sería la de establecer jerarquías. Es decir, la imposibilidad de establecer la primacía de unas formas de conocimiento, de prácticas y de contextos de acción por sobre otros, contraria a la destrucción de otras formas de saber.

En vez de orientarse por la acumulación, lo hace por el reconocimiento de su permanente incompletitud; en vez de orientarse por el punto de llegada (la predictibilidad), lo hace por el punto de partida; es decir, por las condiciones bajo las cuales diversas racionalidades y sujetos están en condiciones de argumentar; por lo tanto, al determinismo de la ciencia reguladora se opone el relativismo de las circunstancias contextuales. Sin embargo, no es un relativismo acrítico, sino uno sustentado en al menos dos principios básicos: el respeto de la dignidad humana y la posibilidad de discusión democrática (Santos, 1998a). Esta es la diferencia fundamental con la posmodernidad celebratoria.

El conocimiento tampoco puede ser lineal, pues eso negaría otros cursos de acción posibles que deben ser considerados en este proceso horizontal de construcción del conocimiento. Implica, además, la aceptación y el reconocimiento de escalas temporales y espaciales que pierden visibilidad frente a formas dominantes de orden y progreso modernas-occidentales, las cuales tienden constantemente a interpretaciones universalistas. Santos (1998a: 434) señala que "el conocimiento en el nuevo paradigma es tan temporal como las prácticas y la cultura a las que se vincula. Asume plenamente su carácter incompleto, debido a que siendo un conocimiento presente, sólo permite la inteligibilidad del presente".

Esta incompletitud del conocimiento debe vincularse con los espacios estructurales de acción (Santos, 2003). Implica reconocer que se ha privilegiado acumular conocimiento sobre ciertos fenómenos en determinadas 
escalas, y que no hemos visto o que hemos silenciado saberes y fenómenos sociales en otras. Se ha prestado mayor atención a cuestiones de orden económico o político, y la aplicación de marcos epistemológicos de esas escalas a problemas situados en el ámbito identitario o de relaciones de género o intergeneracionales, que en realidad responde a patrones de conocimiento completamente diferentes.

Por tanto, lo que plantea Santos es una inversión del orden dominante, al menos hasta la superación de la crisis y la aparición de un nuevo equilibrio dialéctico. Este nuevo equilibrio dialéctico a favor de la emancipación debe orientar las acciones de las ciencias sociales críticas.

El medio para salir de este impasse en un contexto de transición paradigmática consiste en revalorizar el conocimiento-emancipación, dándole la primacía sobre el conocimiento-regulación. Esto implica, por un lado, que la solidaridad se transforme en la forma hegemónica de saber, y, por otro, que la positividad del caos sea reconocida en cuanto parte integrante del orden solidario (Santos, 2009: 64).

Cabe preguntarse si el hecho de decantarse por un conocimiento-emancipación es una situación permanente o más bien una necesidad transitoria producto de la crisis aquí descrita, y que podría requerir en el futuro una nueva búsqueda de equilibrio. En la medida en que Santos opta por una visión posmoderna de la ciencia, una que no cree en las certezas, en las visiones inequívocas, en las leyes permanentes, nos preguntamos: ¿la opción por la emancipación está asociada a la crisis del capitalismo y la ciencia o es una opción permanente que debe estar presente en los distintos órdenes sociales? Frente a estas cuestiones, Santos (2003: 380-381) señala que:

A medida que la transición paradigmática progresa, las luchas emancipadoras dejarán de combatir las formas de regulación social que ahora existen para combatir las nuevas formas de regulación surgidas, entretanto, de las propias luchas emancipadoras paradigmáticas. Esta vigilancia permanente sobre sí misma y su autorreflexividad distinguen la emancipación posmoderna de la emancipación moderna.

Así planteada la cuestión, la Epistemología del Sur implica abrir paso creativamente a nuevas formas de organización y de conocimiento social, a partir de un diagnóstico crítico del presente, para construir un mundo más justo y libre. En el siguiente apartado proponemos una discusión sobre los desafíos que implica avanzar por esta vía hacia una nueva práctica metodológica.

\section{Ausencia y emergencia: bases para el desarrollo de problemas de investigación}

La generación de conocimiento-emancipación requiere formas de acceso, encuentro y diálogo con el mundo social en diversas escalas y ámbitos sociales, 
orientados a recuperar el equilibrio entre la regulación y la emancipación. Para ello se requiere una actitud reflexiva sobre las prácticas de encuentro, diálogo y producción de conocimiento que busquen activamente evitar que la generación de conocimiento se convierta en regulación social.

En las siguientes páginas se discutirá sobre tres orientaciones generales hacia el conocimiento- emancipación: la sociología de las ausencias y emergencias como punto de partida para problematizar lo social; la producción ecológica de saber (en un contexto de múltiples ecologías); y la traducción intercultural como desafío para el análisis y sistematización del conocimiento.

La intención no es producir una receta metodológica rígida, sino la identificación de elementos que se pueden mezclar y adaptar a partir de necesidades contextualizadas de generación de conocimiento. Examinamos en primer lugar los desafíos para la observación empírica que provienen de la sociología de las ausencias y de las emergencias.

Las preocupaciones, los nuevos problemas que orientan la producción de saber, surgen desde la necesidad de ampliar el presente y contraer el futuro. Según Santos (2005), tanto la sociología de las ausencias como la sociología de las emergencias parten del inconformismo ante el desperdicio de experiencia y ante la carencia de posibles futuros emancipatorios, respectivamente.

Por ello, no es posible pensar en el agotamiento de las fuentes de investigación social. La dificultad para las interpretaciones críticas radica más bien en nuestra incapacidad de ampliar la mirada y desocultar múltiples casos diseminados en el mundo social como un ejercicio paralelo y en oposición a la ciencia convencional.

Es decir, una búsqueda de dar valor a experiencias sociales hasta el momento postergadas o negadas, siendo la primera vía de acceso; la búsqueda de aquello que ha sido producido como ausente "intenta demostrar que lo que no existe es, en verdad, activamente producido como no existente, esto es, como una alternativa no creíble a lo que existe [...] el objetivo de la sociología de las ausencias es transformar objetos imposibles en posibles, y basándose en ellos transformar las ausencias en presencias" (Santos, 2005: 160). La diversificación de objetos de estudio responde al ejercicio de la sociología de las ausencias, acusando el cotidiano desperdicio de experiencia social: "El ejercicio de la sociología de las ausencias es contrafáctica y tiene lugar a través de una confrontación con el sentido común científico tradicional. Para ser llevada a cabo, exige imaginación sociológica” (Santos, 2005: 166).

A través de la referencia al concepto desarrollado por Wright Mills (1987), se entiende que la búsqueda de las ausencias es una cualidad mental que implica pensar agudamente en fenómenos olvidados, y por medio de un 
ejercicio crítico, desentramar sus características primordiales. En otra parte, aunque apuntando a la misma idea de fondo, habla de una perspectiva curiosa: "[...] entiendo la búsqueda de un ángulo diferente a partir del cual las proporciones y las jerarquías establecidas por la perspectiva normal puedan ser desestabilizadas, y, consecuentemente, ver subvertida su pretensión de una representación de la realidad ordenada y fiel” (Santos, 2009: 93).

Santos (2003) plantea esa búsqueda de lo olvidado como una tarea de excavación arqueológica en aquellos lugares donde nada ha sido estudiado. Las reflexiones teóricas cumplen un rol de acompañamiento y de diálogo con las experiencias sociales que se vuelven centrales (Ziai, 2013).

La segunda herramienta para la problematización de lo social es la emergencia, pues permite comprender capacidades y potencialidades en experiencias sociales habitualmente no tomadas en cuenta. Es, por tanto, un ejercicio para buscar la utopía en la experiencia social: "La sociología de las emergencias consiste en la investigación de las alternativas que caben en el horizonte de las posibilidades concretas" (Santos, 2005: 169).

Esto orienta la búsqueda, identificación y promoción de las alternativas al proyecto hegemónico de la modernidad capitalista. Su objetivo es generar futuros de posibilidades, utópicas y realistas, a partir de las capacidades y potencialidades del presente. Utópicas en tanto expanden el campo de las experiencias sociales posibles y se comprometen con un futuro que no es inmediato, sino que es pensado como la ampliación simbólica de pistas o señales alternativas en un futuro distante. Realistas, en la medida en que invitan al diálogo argumentativo con experiencias sociales que ya existen.

El campo de la sociología de las emergencias es el campo de las expectativas utópicas, el campo de la sociología de las ausencias es el de las experiencias que afloran en los intersticios de los proyectos hegemónicos, especialmente aquel espacio de las sociedades actuales que, de acuerdo con Santos, ha resistido mejor a la regulación social: la comunidad.

\section{La ecología de saberes en un contexto de múltiples ecologías}

La ecología es entendida por Santos (2009: 113) como "la práctica de agregación de la diversidad a través de la promoción de interacciones sustentables entre entidades parciales y heterogéneas". Existen diversos tipos de ecologías: la de saberes, la de las temporalidades para trascender la monocultura del tiempo lineal, la del reconocimiento que se revela al colonialismo, la de la productividad, que estudia formas alternativas de producción, y la de las transescalas. Destacamos acá la relevancia de dos miradas ecológicas: saberes y escalas. 
La ecología de saberes constituye la multiplicación y diversificación de las experiencias disponibles gracias a la sociología de las ausencias. A través de la ecología de saberes se pretende hacer un uso contrahegemónico de la ciencia hegemónica, acercándola a otras formas de saberes, laicos, con el saber popular, con el saber indígena, el saber urbano popular o el saber campesino.

La ecología de saberes consiste en otorgar igualdad de condiciones a las distintas formas de saber como "forma epistemológica de las luchas sociales emancipatorias emergentes sobre todo en el Sur" (Santos, 2009: 117). La ecología de saberes implica además la búsqueda de saberes marginados y prácticas marginadas, con la necesidad de revalorizar el conocimiento aplicado a una práctica, a una forma de vivir desde una experiencia silenciada o emergente contra los proyectos hegemónicos; y, como principio epistemológico exige el ejercicio de exploración arqueológica a través del estudio de saberes y prácticas producidos constantemente como no inexistentes.

Por otra parte, la ecología de las transescalas permite trascender las distinciones entre lo local y lo global, al mismo tiempo que posibilitan la organización institucional de una serie de dimensiones o espacios de la vida social.

Esta consideración ecológica de las escalas y dimensiones de la vida social contribuyen a una comprensión de la sociedad que busca superar la lógica habitual entre estructura y acción (Santos, 2003: 311). La investigación se orienta hacia la forma en que el poder, el derecho y el conocimiento emergen y cruzan entre estas dimensiones o espacios estructurales: 1) el espacio doméstico, 2) el espacio de producción, 3) el espacio de mercado, 4) el espacio de comunidad, 5) el espacio de la ciudadanía y 6) el espacio mundial.

En cada uno de ellos se expresan las asimetrías de las sociedades modernas, que se manifiestan específicamente como diferencias en prácticas sociales organizadas institucionalmente por medio de formas de poder, derecho y conocimiento, poniéndose de manifiesto en diversas prácticas sociales y procesos de interacción social. Un mismo fenómeno puede atravesar más de una dimensión manifestándose tanto local como globalmente. Aquella transformación que ocurre en los macroespacios impacta y se fija en los espacios locales se constituye en un globalismo localizado; pero al mismo tiempo, lo local puede transcender y afectar lo global, convirtiéndose en localismo globalizado (Santos, 2003). Esta ecología de las transescalas parte:

de un modelo analítico que identifica los principales procesos de estructuración y de práctica social, constelaciones de relaciones que aseguran, en conjunto, el sentido y el ritmo de la transformación social o el bloqueo de esta [...] este modelo se puede aplicar tanto a las sociedades nacionales como a las sociedades subnacionales y a las sociedades transnacionales (Santos, 2003: 375-376). 
Existe, por tanto, una precaución inicial a nivel metodológico a tener en cuenta por los investigadores que estudian fenómenos sociales con expresión en distintas escalas. Un error habitual en ciencias sociales es la sobreposición o el estudio de fenómenos sociales, confundiendo las escalas en su observación. Un mismo fenómeno social puede dar paso a distintas preguntas de investigación de acuerdo con la escala en que estemos situados; por tanto, cambiar el objeto de estudio. Santos (2003: 67) nos dice lo siguiente: "Las diferencias de escala no sólo son cuantitativas, sino también cualitativas. Un fenómeno dado sólo puede ser representado en una escala dada. Cambiar de escala implica cambiar de fenómeno. Cada escala representa un fenómeno y distorsiona o esconde otros".

La enseñanza común a todas las ecologías es el hecho de que no podemos quedarnos con lo ya conocido, sino que debemos ir más allá, haciendo creíbles otra prácticas y saberes marginados, haciendo frente "al sentido común científico tradicional" (Santos, 2005: 166).

\section{Sobre la traducción de saberes, prácticas y sus procedimientos}

Finalmente, el procedimiento más desafiante de esta dimensión práctica de la propuesta de Santos es la traducción intercultural, en la medida que busca romper tanto con la crisis de la modernidad, como con el nihilismo de la posmodernidad celebratoria. En este sentido general, en el cual se definen las razones para buscar procedimientos alternativos a los dominantes, es que se entiende la traducción como una búsqueda de nuevas formas de emancipación social: esto es, una búsqueda activa de alternativas al proyecto neoliberal.

Santos (2005) procura precisar dos momentos del proceso de traducción: los cuidados epistemológicos y los cuidados en relación con los procedimientos de traducción. Respecto a la epistemología, identifica la traducción entre saberes, así como también la traducción entre prácticas y agentes. La primera de ellas es denominada hermenéutica diatópica y "consiste en un trabajo de interpretación entre dos o más culturas con el objetivo de identificar preocupaciones isomórficas entre ellas y las diferentes respuestas que proporcionan" (Santos, 2005: 175-176). La hermenéutica sobre una diatopía implica la comprensión de las variaciones de las ideas o de formas de saber entre diferentes culturas, y la isomorfía implica la búsqueda de preocupaciones comunes, aunque en la práctica tengan formas diferentes.

Algunos ejercicios de traducción planteados por el intelectual portugués han buscado distinguir las formas que toman en diferentes culturas las preocupaciones por la dignidad humana, las preocupaciones en relación con la 
vida productiva, y la preocupación por la sabiduría y el desarrollo de ver el mundo. En Occidente, por ejemplo, la forma de interpretar la dignidad humana es mediante el concepto de derechos humanos, la vida productiva a través del desarrollo capitalista, y la sabiduría por medio de la filosofía occidental, y que tienen formas de manifestarse como saberes muy diferentes en otras culturas. Por ello, el punto de partida es reconocer la incompletitud de la propia cultura no como un relativismo cultural, sino como un universalismo negativo; ninguna cultura puede ser completa (Santos, 2005: 178).

En cuanto a la traducción entre prácticas y agentes, se busca crear procesos de comprensión recíproca entre formas de organización y objetivos de acción social o política. Estas traducciones entre prácticas se vinculan con experiencias de saber fuertemente diferenciadas como las que pueden existir entre la medicina moderna y la tradicional; aunque también pueden ocurrir entre prácticas similares, pero que por razones culturales o históricas no se han reconocido como tales, como lo que podría suceder entre un movimiento feminista y un movimiento indígena o ecológico.

Un elemento clave para la definición consensuada y democrática de procedimientos de traducción es la noción de zona de contacto, es decir, "campos sociales donde diferentes mundos de vida normativos, prácticas y conocimientos se encuentran, chocan e interactúan” (Santos, 2005: 181).

Las zonas de contacto surgen entonces a partir de experiencias concretas e históricas de sujetos sociales, que debido a la sociología de las ausencias y de las emergencias se manifiestan como existentes, ya sea como encuentros de imposición colonial o hegemónica o como el encuentro de actores subalternos o contrahegemónicos. Dependiendo de ese punto de partida, y de la forma como se desarrolle el proceso de traducción, puede irse ampliando o reduciendo una relación de confianza entre agentes, lo cual posibilita traer a la discusión nuevos saberes y prácticas.

Uno de los factores clave en este proceso de construcción de confianza es el reconocimiento de que los actores involucrados son contemporáneos, pero su forma de experimentar el tiempo y el ritmo de las conversaciones es diverso. Habitualmente en las ciencias sociales, el ritmo de las conversaciones las define el investigador (o la agencia que lo financie); en los procedimientos de traducción son definidos consensualmente, y el ritmo de las conversaciones es uno de esos factores por acordar.

Una de las dimensiones procedimentales más complejas del proceso de traducción planteado por Santos (2005: 184), es la pregunta de quién traduce. Inicialmente, los que deben traducir son los mismos actores sociales: "Los traductores de culturas deben ser intelectuales cosmopolitas. Pueden 
encontrarse tanto entre los dirigentes de movimientos sociales como entre activistas de base". Se entiende que la complejidad proviene de que, al menos, en principio, el rol de los científicos sociales desaparece, en la medida en que el proceso de argumentación y diálogo recíproco no incluye a terceros como observadores objetivos del encuentro intercultural.

Sin embargo, puede entenderse también como un llamado activo a los cientistas sociales a involucrarse más activamente en las luchas sociales de la actualidad y a participar en el procedimiento de traducción desde su militancia cultural, social o política particular.

\section{Abriendo la discusión... para finalizar}

Inicialmente se proponía que la revisión de la obra de Santos implica tres desafíos fundamentales para las ciencias sociales, a partir de los cuales se ha organizado la discusión. El primero de ellos se refiere a la comprensión de las sociedades actuales, sus actores y sus procesos que se manifiestan en escalas locales, nacionales y globales.

Ello implica reconocer que las discusiones teóricas en relación con la modernidad, la posmodernidad, el capitalismo y la globalización (no sólo la económica), son procesos que todavía requieren renovadas interpretaciones teóricas y que suponen un desafío para las ciencias sociales.

La propuesta de Santos para repensar las ciencias sociales parece fundamental para el caso de América Latina; en primer lugar, para pensar nuestro continente transescalarmente, lo cual implica que, en este contexto de transición y crisis social, debemos reflexionar insistentemente respecto a las diversas manifestaciones de desigualdad y exclusión.

Sin embargo, existe una tendencia analítica a fragmentar la crisis concentrándose en las dimensiones globales, económicas y políticas del problema, y prestando menor atención precisamente a aquellos espacios sociales donde la resistencia a los proyectos sociales y políticos capitalistas puede escapar de los límites regulatorios: la comunidad, la vida privada y, como indicábamos con anterioridad, la experiencia subjetiva individual.

Esto implica también que las ciencias sociales latinoamericanas puedan generar aportes para la transformación de todos los espacios sociales de acción. No basta con relaciones internacionales donde el Norte y el Sur se encuentren en equilibrio, no basta con un Estado más profundamente democrático ni tampoco con relaciones productivas y experiencias de consumo justas, si esas transformaciones no van acompañadas de relaciones identitarias marcadas por el reconocimiento solidario de las diferencias, o por relaciones familiares que dejen atrás el patriarcalismo y el sexismo. 
Esto implica prestar especial atención a lo que Santos denomina cambios subparadigmáticos, en la medida en que no están orientados a la emancipación social, considerando todas las escalas de acción y todas las dimensiones institucionales de una sociedad que no puede pensarse sólo como local o nacional, sino también como global.

Ello nos lleva al segundo desafío, el cual implica abrir la discusión epistemológica desde el diálogo intercultural, recuperando la crítica a la racionalidad occidental y al eurocentrismo; es decir, es una invitación para que las ciencias sociales se dejen interpelar por las formas de saber y los conocimientos de los pueblos del mundo como manifiestaciones (ausentes y emergentes) de proyectos utópicos plurales. En este sentido, creemos que sería relevante avanzar en el diálogo entre saberes, tanto de aquellos surgidos desde las experiencias del espacio privilegiado de la comunidad, como de los saberes con pretensiones teóricas generales o universales.

Otra de las cuestiones claves señaladas por Santos tiene que ver con la permanente búsqueda de equilibrio entre formas de conocimiento. Una de las constantes amenazas para el pensamiento crítico y para la búsqueda de emancipación social es la tendencia moderna a fijar y, por tanto, a establecer nuevos o renovados mecanismos de regulación.

Es indispensable avanzar en las estrategias para evitar que toda forma de conocimiento y de acción, en cualquier escala de acción, se constituya con el tiempo en un proyecto hegemónico incuestionable. En el contexto latinoamericano, donde los proyectos políticos de diversos países de la región se ven como modelos de emancipación social, el cuidado respecto a esta búsqueda prudente de emancipación que evita atarse permanentemente a un proyecto de sociedad parece fundamental.

Finalmente, el tercer y último desafío es el de las opciones metodológicas y su intencionalidad práctica orientada a la transformación de la sociedad. Hemos podido apreciar que la propuesta de Santos nos permite ver de nuevo y apreciar mejor nuestra realidad social, en esa búsqueda que debería ser fundamental para las ciencias sociales, de ampliar el presente reconociendo y valorando aquello que los actores y discursos dominantes reducen al olvido, redescubriendo la necesidad del diálogo entre saberes diversos, y el desarrollo de mecanismos prácticos de diálogo, reconocimiento y transformación de la sociedad.

Sabemos que es un reto enorme en nuestras sociedades latinoamericanas, pues pese a que una de las riquezas del continente es su diversidad cultural (la de sus pueblos indígenas, la de los afrodecendientes, la de sus culturas populares), éste sufre la constante tensión de su inferiorización y falta de reconocimiento. 
El desafío epistemológico y metodológico de las ciencias sociales en América Latina es precisamente pasar de una relación con objetos de investigación a una relación con actores sociales, lo cual implica también el reto de legitimar sus proyectos, sus sentidos de acción (que escapan a la racionalidad occidental) y sus principios valórico-normativos, los cuales, a su vez, rompen con la lógica universalista. Esto implica el desafío imaginativo de no absolutizar una única mirada, una única voz.

Sin embargo, una de las principales dificultades a las cuales nos enfrentamos es la enorme fragmentación de las luchas sociales. Hay que recorrer un camino desde los espacios identitarios cerrados, desde las luchas subparadigmáticas profundamente marcadas por la hegemonía capitalista, a un proceso de aprendizaje de las experiencias sociales que tienda al cosmopolitismo, reconozca la permanente incompletitud de nuestros saberes y experiencias locales, y permita el intercambio en comunidades argumentativas de traducción, reguladas por la democracia y el respecto de la dignidad humana.

\section{Referencias}

Aguiló, Antoni (2011), "Boaventura de Sousa Santos: Une proposition du dialogue interculturel à l'époque de la mondialisation", en Revista Utopia y Praxis, vol. 16, núm. 54, Venezuela: Universidad de Zulia. Disponible en: http://goo.gl/DvlP63 [27 de noviembre de 2013].

Álvarez, Luciana (2010), “Más allá del multiculturalismo: Crítica de la universalidad (concreta) abstracta”, Revista Unisinos, vol. 11, núm. 2, Brasil: Universidade do Vale do Rio dos Sinos.. Disponible en: http://goo.gl/7JLev3 [27 de noviembre de 2013].

Anibal, Graça (2010), “A Teoria Crítica e a Educação”, en Revista Lusófona de Educação, vol. 16, núm. 16, Portugal: Universidade Lusófona de Humanidades e Tecnologias. Disponible en: http://goo.gl/z0wVzN [27 de noviembre de 2013].

Avritzer, Leonardo (2002), "Towards a pattern of world citizenship", en Lua Nova: Revista de Cultura e Política, núm. 55-56, Brasil: Centro de Estudos de Cultura Contemporânea. Disponible en: http://goo.gl/RecfpV [28 de junio de 2012].

Baker, Michael (2012), "Modernity/coloniality and Eurocentric education: Towards a postoccidental self-understanding of the present", en Policy Futures in Education, vol. 10, núm. 1. Estados Unidos de América: SAGE. Disponible en: http://pfe.sagepub.com/ content/10/1/4.full.pdf + html [28 de noviembre de 2013].

Barreto, José-Manuel (2014), "Epistemologies of the south and human rights: Santos ad the quest for global and cognitive justice”, en Indiana Journal of Global Legal Studies, núm. 21, Estados Unidos de América: Indiana University Press. Disponible en: https://goo. gl/uMQXWH [30 de junio de 2015].

Darian-Smith, Eve (1998), "Power in Paradise: The Political Implications of Santos's Utopia”, en Law \& Social Inquiry, vol. 23, núm. 1, Estados Unidos: Wiley \& American bar Foundation. Disponible en: http://goo.gl/nDNVyA [28 de noviembre de 2013]. 
Helder Binimelis-Espinoza y Andrés Roldán-Tonioni. Sociedad, epistemologia y metodología en Boaventura de Sousa Santos

Guarino, Carmela (2009), "Emancipazione e diritti umani nel pensiero di Boaventura de Sousa Santos", en Revista Utopía y Praxis, vol. 14, núm. 46, Venezuela: Universidad de Zulia. Disponible en: http://goo.gl/iDGzRg [28 de noviembre de 2013].

Gutierrez, Daniela (2013), "Boaventura de Sousa Santos and the intercultural reconstruction of human rights”, en Jurídicas, núm. 9, Colombia: Universidad de Caldas. Disponible en: http://goo.gl/xoBKN [30 de junio de 2015].

Machado, Carlos et al. (2012), "Uma aproximação teórica entre os postulados do ideal de sustentabilidade e a sociologia das ausências de Boaventura Sousa Santos", en Revista Brasileira de Gestão e Desenvolvimento Regional, vol. 8, núm. 1, Brasil: Universidade de Taubaté. Disponible en: http://arca.icict.fiocruz.br/handle/icict/6473 [28 de noviembre de 2013].

Marques, Luciana (2008), "Democracia radical e democracia participativa: contribuições teóricas à análise da democracia na educação”, en Educação \& Sociedade, vol. 29, núm. 102, Brasil: Centro de Estudos Educação e Sociedade. Disponible en: http://goo.gl/ RWL1bC [28 de noviembre de 2013].

Mills, C. Wright (1987), La Imaginación sociológica, México: Fondo de Cultura Económica.

Nascimento, Marlene et al. (2013), "The medical rationale category and a new epistemology in health”, en Ciênc. Amp Saúde Coletiva, núm. 18, Brasil: Associação Brasileira de Saúde Coletiva. Disponible en: http://goo.gl/0tKwJx [30 de junio de 2015].

Paredes, Juan (2014), "Pensamiento epistémico y conocimiento social: emergencias y potencialidades en la investigación social", Revista de Estudios Sociales, núm. 48, Colombia: Universidad de los Andes \& Fundación Social. Disponible en: http://goo. gl/jP2gfs [30 de junio de 2015].

Passos, Mailsa (2014), "Encontros cotidianos e a pesquisa em Educação: relações raciais, experiência dialógica e processos de identificação”, en Educar Em Revista, vol. 30, núm. 51, Brasil: Universidade Federal do Paraná. Disponible en: http://goo.gl/AL26GX [30 de junio de 2015].

Pereira, Marcus y Carvalho, Ernani (2008), "Boaventura de Sousa Santos: por uma nova gramática do político e do social”, en Lua Nova: Revista de Cultura e Política, núm. 73, Brasil: Centro de Estudos de Cultura Contemporânea. Disponible en: http://goo.gl/ KXX6VY [28 de noviembre de 2013].

Said, Edward (2007), Orientalismo, España: DeBolsillo.

Santos, Boaventura de Sousa (1998a), De la mano de Alicia: Lo social y lo politico en la postmodernidad, Colombia: Siglo del Hombre Editores, Ediciones Uniandes.

Santos, Boaventura de Sousa (2003), Critica de la Razón Indolente. Contra el desperdicio de la experiencia, España: Desclée de Brouwer.

Santos, Boaventura de Sousa (2005), El milenio huérfano. Ensayos para una nueva cultura politica, España: Trotta.

Santos, Boaventura de Sousa (2006), A gramática do tempo: para uma nova cultura politica, Brasil: Cortez Editora.

Santos, Boaventura de Sousa (2009), Una epistemología desde el Sur, México: CLACSO y Siglo XXI.

Santos, Boaventura de Sousa (2010), Refundación del estado en América Latina: perspectivas desde una epistemología del sur, México: Siglo XXI. 
Santos, Boaventura de Sousa (1998b), "Oppositional Potmodernism and Globalizations: [Commentary]”, en Law \& Social Inquiry, vol. 23, núm. 1, Estados Unidos: Wiley \& American bar Foundation. Disponible en: https://goo.gl/PIH6kw [28 de noviembre de 2013].

Santos, José dos et al. (2015), "A mundialização da sociologia contemporânea: diálogos entre as sociologias na América Latina, na Índia e na China”, en Sociedade e Estado, núm. 30, Brasil: Universidade de Brasilia. Disponible en: http://goo.gl/u0p375 [30 de junio de 2015].

Sarmento, Clara (2015), "Writing and Living on the Stage of History: Women and Intercultural Transits between Portugal and Brazil in the Early Nineteenth Century", en Hispanic Research Journal-Iberian and Latin American Studies, núm. 16, Reino Unido: Taylor \& Francis. Disponible en: http://goo.gl/pfZH4L [30 de junio 2015].

Sieder, Rachel (2011), “Emancipation' or 'regulation'? Law, globalization and indigenous peoples' rights in post-war Guatemala”, en Economy and Society, vol. 40, núm. 2, Reino Unido: Taylor \& Francis. Disponible en: http://goo.gl/OsZk0p [28 de noviembre 2013].

Streck, Danilo y Adams, Telmo (2012), "Pesquisa em educação: os movimentos sociais e a reconstrução epistemológica num contexto de colonialidade”, en Educacao e Pesquisa, vol. 38, núm. 1. Brasil: Faculdade de Educação da Universidade de São Paulo. Disponible en: http://goo.gl/sxmmwQ [28 de noviembre de 2013].

Tamayo, José (2011), "Boaventura de Sousa Santos: hacia una sociología de las ausencias y las emergencias", en Utopia y Praxis latinoamericana: revista internacional de filosofía iberoamericana y teoría social, núm. 54, Venezuela: Universidad de Zulia. Disponible en: http://goo.gl/mQuJCG [28 de noviembre de 2013].

Umut, Ziya y Ballestero, Andrea (2014), "Regulatory Translations: Expertise and Affect in Global Legal Fields", en Indiana Journal of Global Legal Studies, núm. 21, Estados Unidos de América: Indiana University Press. Disponible en: https://goo.gl/QyRSNq [30 de junio de 2015].

Villarreal, José (2006), “Subjetividad, Ciudadanía y Emancipación. Sobre el capítulo noveno del libro De la mano de Alicia. Lo social y lo político en la posmodernidad”, en Tabula Rasa, núm. 5, Colombia: Universidad Colegio Mayor de Cundinamarca. Disponible en: http://ref.scielo.org/d8mg8m [30 de junio de 2015].

Ziai, Aram (2013), "Boaventura de Sousa Santos", en Development and Change, vol. 44, núm. 3, Estados Unidos de América: Wiley. Disponible en: http://goo.gl/gJ2FPe [30 de junio de 2015].

Helder Binimelis-Espinoza. Doctor en Investigación en Ciencias Sociales con mención en sociología por FLACSO, México. Magíster en Estudios Sociales y Políticos Latinoamericanos, Licenciado en Comunicación Social, Universidad Católica de Temuco, Departamento de Trabajo Social. Líneas de Investigación: Teoría crítica de la tecnología, propiedad intelectual. Publicaciones recientes: Binimelis-Espinoza, Helder, "Brasil y Chile: diversas estrategias de integración comercial, diferentes leyes y políticas de propiedad 
industrial”, en Vniversitas, vol. 64, núm. 131 (2015). Disponible en: http:// goo.gl/WkzNGE; Binimelis-Espinoza, Helder, "La experiencia personal y el diálogo teórico como insumos para el desarrollo de un problema de investigación”, en Zaremberg, Gisela et al. [eds.], El helicoide de la investigación: metodología en tesis de ciencias sociales, México: FLACSO (2013); BinimelisEspinoza, Helder, "Contrapuntos críticos a la Sociedad del Conocimiento", en Revista Gestión de las Personas y Tecnología, noviembre (2010). Disponible en: http://goo.gl/ir5Ayd

Andrés Roldán-Tonioni. Licenciado en Sociología en la Universidad de la Frontera, Magíster en Psicología Comunitaria de la Universidad de Chile, Doctorando en Ciencias Sociales en la Universidad de Chile. Actualmente es profesor de Investigación Social para las carreras de Trabajo Social en las Universidades Andrés Bello y Universidad Cardenal Silva Henrríquez, Chile. Líneas de investigación: democracia participativa, teoría crítica, ciudadanía y sociedad civil. Publicaciones recientes: Roldán, A. y Rubilar, G., "Áreas de Desarrollo Indígena: Estudio de caso del ADI Puel Nahuelbuta, como estrategia de las políticas públicas en el mundo mapuche", en Revista Universum, vol. 2, núm. 29 (2014). Disponible en: http://goo.gl/lFcEiC; Roldán, A., "Los oficios tradicionales en la modernidad", en Revista de Trabajo Social Perspectivas, vol. XVI, núm. 22 (2011). Disponible en: http://goo.gl/aVJePA; Roldán, A., "Prácticas comunitarias del desarrollo. Una mirada desde los oficios y el trabajo”, en Polis, vol. 8, núm. 24 (2009). Disponible en: http:// goo.gl/sXPG1F.

Recepción: 16 de noviembre de 2016.

Aprobación: 10 de mayo de 2017. 
